\title{
Comparative integromics on BMP/GDF family
}

\author{
YURIKO KATOH $^{1}$ and MASARU KATOH ${ }^{2}$ \\ ${ }^{1}$ M\&M Medical BioInformatics, Hongo 113-0033; ${ }^{2}$ Genetics and Cell Biology Section, \\ National Cancer Center Research Institute, Tokyo 104-0045, Japan \\ Received January 18, 2006; Accepted February 23, 2006
}

\begin{abstract}
WNT, Notch, FGF, Hedgehog and BMP signaling pathways network together during embryogenesis, tissue regeneration, and carcinogenesis. $B M P 2, B M P 3, B M P 4, B M P 5$, BMP6, BMP7, BMP8A, BMP8B, BMP10, BMP15, AMH, GDF1, GDF2, GDF3, GDF5, GDF6, GDF7, GDF8, GDF9, $G D F 10, G D F 11$, and $G D F 15$ are $B M P / G D F$ family genes within the human genome; however, transcriptional regulation of $B M P / G D F$ family members by the canonical WNT signaling pathway remains unclear. We searched for the TCF/LEF-binding site within the promoter region of $B M P / G D F$ family genes by using bioinformatics and human intelligence. Because four TCF/LEF-binding sites were identified within human GDF 10 promoter, comparative genomics analyses on GDF10 orthologs were further performed. Chimpanzee GDF10 gene, encoding a 477amino-acid protein, was identified within NW_112875.1 genome sequence. AY412135.1 was not the correct coding sequence for chimpanzee GDF10. Chimpanzee GDF10 showed $99.2 \%, 83.2 \%$ and $47.4 \%$ total amino-acid identity with human GDF10, mouse Gdf10 and human BMP3, respectively. RASGEF1A-GDF10-PRKG1 locus at human chromosome 10q11 and BMP3-PRKG2-RASGEF1B locus at human chromosome $4 \mathrm{q} 21$ were paralogous regions with insertions/deletions and recombination. Human GDF10 mRNA was expressed in fetal cochlea, fetal lung, testis, retina, pineal gland, other neural tissues, head and neck tumors, while mouse Gdf10 mRNA was expressed in fetal liver, inner ear, cerebellum, other neural tissues, prostate and blood vessels. Four TCF/LEF-binding sites in human GDF10 promoter were conserved in chimpanzee GDF10 promoter, but not in the mouse Gdf10 promoter; however, another TCF/LEF-binding site occurred in mouse Gdf10 promoter. Four bHLH-binding sites in human GDF 10 promoter were conserved in chimpanzee GDF10 promoter, but only one in mouse Gdf10
\end{abstract}

Correspondence to: Dr Masaru Katoh, Genetics and Cell Biology Section, National Cancer Center Research Institute, 5-1-1 Tsukiji, Chuo-ku, Tokyo 104-0045, Japan

E-mail: mkatoh@ncc.go.jp

Key words: bioinformatics, comparative genomics, comparative proteomics, WNT, BMP, GDF, integrome network, systems medicine promoter. Primate $G D F 10$ promoters were divergent from mouse Gdf10 promoter. Because GDF10 was characterized as a potential target of canonical WNT signaling pathway in neural tissues, GDF10 is one of the targets of systems medicine, especially in the field of regenerative medicine.

\section{Introduction}

WNT, Notch, FGF, Hedgehog, and BMP signaling pathways network together during embryogenesis, tissue regeneration, and carcinogenesis (1-8). Canonical WNT signals are transduced through Frizzled receptors and LRP5/6 co-receptors to activate transcription of target genes, such as FGF18, FGF20, $D K K 1$ and $D K K 4$, based on the transcriptional complex consisting of TCF/LEF, ß-catenin, BCL9/BCL9L, and PYGO1/ PYGO2 (9-19).

TGFß superfamily members are classified into TFGß/ Nodal/Activin group and BMP/GDF group. TFGß/Nodal/ Activin signals are transduced through type I and type II receptors for each member to R-SMAD proteins, such as SMAD2 and SMAD3, while BMP/GDF signals are transduced through type I and type II receptors for each member to R-SMAD proteins, such as SMAD1, SMAD5 and SMAD8. Phosphorylated R-SMADs associated with SMAD4 are then translocated to the nucleus to activate transcription of target genes (20-24).

BMP2, BMP3, BMP4, BMP5, BMP6, BMP7, BMP8A, $B M P 8 B, B M P 10, B M P 15, A M H$, GDF1, GDF2, GDF3, GDF5, GDF6, GDF7, GDF8, GDF9, GDF10, GDF11, and GDF15 are $B M P / G D F$ family genes within the human genome (http:// Www.gene.ucl.ac.uk); however, transcriptional regulation of $B M P / G D F$ family members by the canonical WNT signaling pathway remains unclear. We searched for the $\mathrm{TCF} / \mathrm{LEF}-\mathrm{binding}$ site within the promoter region of $B M P / G D F$ family genes by using bioinformatics and human intelligence. Because four TCF/LEF-binding sites were identified within human GDF 10 promoter, comparative genomics analyses on GDF 10 orthologs were further performed.

\section{Materials and methods}

WNT target gene screening. Genome sequences corresponding to human $B M P 2, B M P 3, B M P 4, B M P 5, B M P 6, B M P 7$, BMP8A, BMP8B, BMP10, BMP15, AMH, GDF1, GDF2, GDF3, GDF5, GDF6, GDF7, GDF8, GDF9, GDF10, GDF11 
A

\begin{tabular}{|c|c|c|c|c|c|c|c|}
\hline $\begin{array}{c}\text { Human } \\
\text { gene }\end{array}$ & RefSeq ID & $\begin{array}{c}\text { Genome } \\
\text { clone }\end{array}$ & $\begin{array}{c}\text { TCF / LEF - } \\
\text { binding sites }\end{array}$ & $\begin{array}{c}\text { Human } \\
\text { gene }\end{array}$ & RefSeq ID & $\begin{array}{c}\text { Genome } \\
\text { clone }\end{array}$ & $\begin{array}{l}\text { TCF / LEF - } \\
\text { binding sites }\end{array}$ \\
\hline$B M P 2$ & NM_001200.2 & AL035668.15 & 0 & GDF1 & NM_001492.3 & $A C 005197.1$ & 0 \\
\hline ВMP3 & NM_001201.1 & AC093883.2 & 1 & GDF2 & NM_016204.1 & AL.731561.12 & 2 \\
\hline BMP4 & NM_001202.2 & AL138479.4 & 1 & GDF3 & NM_020634.1 & $A C 006927.27$ & 3 \\
\hline BMP5 & NM_021073.2 & AL137178.7 & 2 & GDF5 & NM_000557.2 & AL121586.31 & 0 \\
\hline$B M P G$ & NM_001718.2 & AL135778.9 & 0 & GDFG & NM_001001557.1 & AC015998.8 & 0 \\
\hline BMP7 & NM_001719.1 & AL122058.19 & 0 & GDF7 & NM_182828.2 & $A C 012065.8$ & 1 \\
\hline$B M P 8 A$ & NM_181809.1 & AL365277.23 & 0 & GDFB & NM_005259.1 & $A C 073120.5$ & 0 \\
\hline$B M P 8 B$ & NM_001720.3 & AL033527.26 & 0 & GDFG & NM_005260.2 & AC00 4500.1 & 0 \\
\hline$B M P 10$ & NM_014482.1 & AC097495.3 & 0 & GDF1O & NM_004962.2 & AL731561.12 & 4 \\
\hline BMP15 & NM_005448.1 & AL359914.14 & 0 & GDF11 & NM_005811.2 & $A C 073487.34$ & 0 \\
\hline$A M H$ & NM_000479.2 & $A C 138127.1$ & 0 & GDF15 & NM_004864.1 & $A C 008397.7$ & 1 \\
\hline
\end{tabular}
CACACACGGGGCOCACGCACACGGCAGACGGGCCAGGGACGACCCTGTCAGCTECAGCCCCAAAGGTCCGGGGCGCACAGCCGGGTCCCCTCGAGGTCGCAGCTGGCCGCCCCCGCCCCGCCCCTCGAAGCAGCCGGGCCCGGGCGCGCAGTG

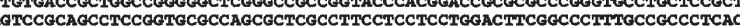

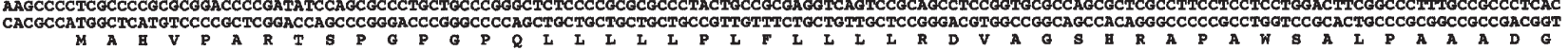

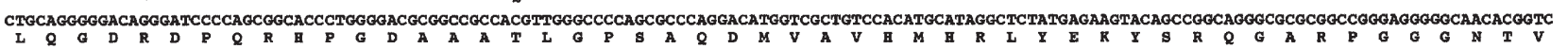
CGCAGCTTCAGGGCCAGGCTGGAAGTGGTCGACCAGAAGGCCGTGTATTTCTTCAACCTEACTTCCATGCAAGACTCGGAAATGATCCTTACGGCCACTTTCCACTTCTACTCAGAGCCGCCTCGGTGGCCTCGAGCGCTCGAGGTGCTA

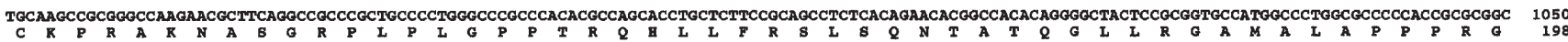
CTGTGGCAGGCCAAGGACATCTCCCCCATCGTCAAGGCGGCCCGCCGGATGGCGAGCTECTCCTCTCCGCCCAGCTGGATTCTGAGGAGAGGGACCCGGGGGTCCCCCAGCCCAGCCCCTATGCGCCCTACATCCTAGTCTATGCCAAC 1200

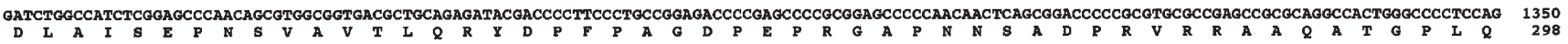

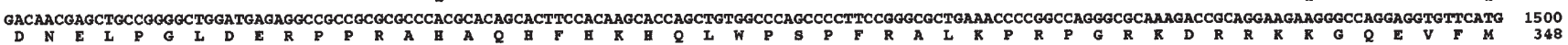
GCCGCCTCGCAGGTGCTGGACTTTGACGAGAAGACGATGCAGAAAGCCCGGAGGAAGCAGTGGGATGAGCCGAGGGTGTGCTCCCGGAGGTACCTGAAGGTGGACTTCGCAGACATCGGCTGGAATGAATGGATAATCTCACCGAAATCT 1650

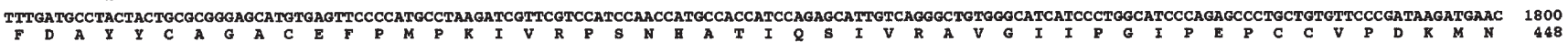
TCCCTTGGGGTCCTCTTCCTGGATGAGAGTCGGAATGTGGTTCTGAAGGTGACCCCAACATGTCCGTGGACACCTGTGCCTGCCGGTGAGACCACTCCAGGGTGGAAAGAAGCCACGCCCAGCAGAGCCGCCTTCTCGGAGCCTTCTGC 1950 AACCAGGACTTGTGGTGCAGCTGCAGACACAGAGCACAGCTCATGGGCAACATCACTGGGGCCCAGAGAGAGCTGTCCGCCAGTGCATCATTAGGGGATCTTTCATTGCTAGTGACTAGCCCCTTAAATGCCAGCCTGAGTACCTGAAGG 2100 CTCCCTGCACACG

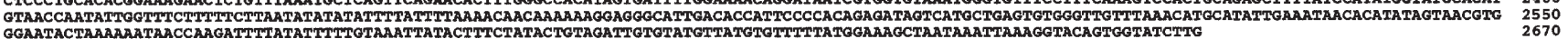

Figure 1. (A), Human $B M P / G D F$ gene family. Genome sequences corresponding to 5'-promoter region of $22 B M P / G D F$ family members are listed up. Four TCF/ LEF-binding sites occur within the GDF10 promoter. (B), Nucleotide and amino-acid sequences of chimpanzee GDF10. Nucleotides and amino-acid residues are numbered on the right.

and GDF15 genes were searched for with BLAST programs (http://www.ncbi.nlm.nih.gov) as described previously (25-28). $\mathrm{TCF} / \mathrm{LEF}$-binding sites within the 5'-flanking promoter region of the above genes were searched for based on bioinformatics and manual inspection as described previously $(29,30)$.

Identification of the chimpanzee GDF10 ortholog. Chimpanzee genome sequences homologous to human GDF10 were searched for with BLAST programs as described previously (31-34). Exon-intron boundaries were determined based on the consensus sequence of exon-intron junctions ('gt ... ag' rule of intronic sequence) and codon usage within the coding region as described previously (35-38). Coding sequence of chimpanzee GDF10 was determined by assembling exonic regions.

Comparative proteomics analysis. Phylogenetic analyses on mammalian BMP/GDF family members were performed by using the CLUSTALW program.

Comparative genomics analyses. Promoter region of mammalian GDF 10 orthologs were aligned by using the Genetyx program and manual curation. TCF/LEF-binding sites within the promoter region were determined as mentioned above.

In silico expression analysis. Expressed sequence tags (ESTs) derived from GDF10 orthologs were searched for by using the BLAST programs. The sources of ESTs were listed up for in silico expression analysis on GDF 10 orthologs.

\section{Results}

Screening of TCF/LEF-binding site within promoter region of BMP/GDF family genes. Human BMP2 Refseq (NM_001200.2), BMP3 Refseq (NM_001201.1), BMP4 Refseq (NM_001202.2), BMP5 Refseq (NM_021073.2), BMP6 Refseq (NM_001718.2), BMP7 Refseq (NM_001719.1), BMP8A Refseq (NM_181809.1), BMP8B Refseq (NM_001720.3), BMP10 Refseq (NM_014482.1), BMP15 Refseq (NM_005448.1), AMH Refseq (NM_000479.2), GDF1 Refseq (NM_001492.3), GDF2 Refseq (NM_016204.1), GDF3 Refseq (NM_020634.1), GDF5 Refseq (NM_000557.2), GDF6 Refseq (NM_001001557.1), GDF7 Refseq (NM_182828.2), GDF8 Refseq (NM_005259.1), GDF9 Refseq (NM_005260.2), GDF10 Refseq (NM_004962.2), GDF11 Refseq (NM_ 005811.2) and GDF15 Refseq (NM_004864.1) were used as query sequences for the BLAST programs to identify genome clones corresponding to $B M P / G D F$ family genes. The 5'flanking promoter region of human $B M P 2, B M P 3, B M P 4$, BMP5, BMP6, BMP7, BMP8A, BMP8B, BMP10, BMP15, $A M H, G D F 1, G D F 2, G D F 3, G D F 5, G D F 6, G D F 7, G D F 8$, GDF9, GDF10, GDF11 and GDF 15 genes were identified within AL035668.15, AC093883.2, AL138479.4, AL137178.7, AL135778.9, AL122058.19, AL365277.23, AL033527.26, AC097495.3, AL359914.14, AC138127.1, AC005197.1, AL731561.12, AC006927.27, AL121586.31, AC015998.8, AC012065.8, AC073120.5, AC004500.1, AL731561.12, AC073487.34 and AC008397.7 genome sequences, respectively (Fig. 1A). TCF/LEF-binding sites within the 5'-promoter region 
A

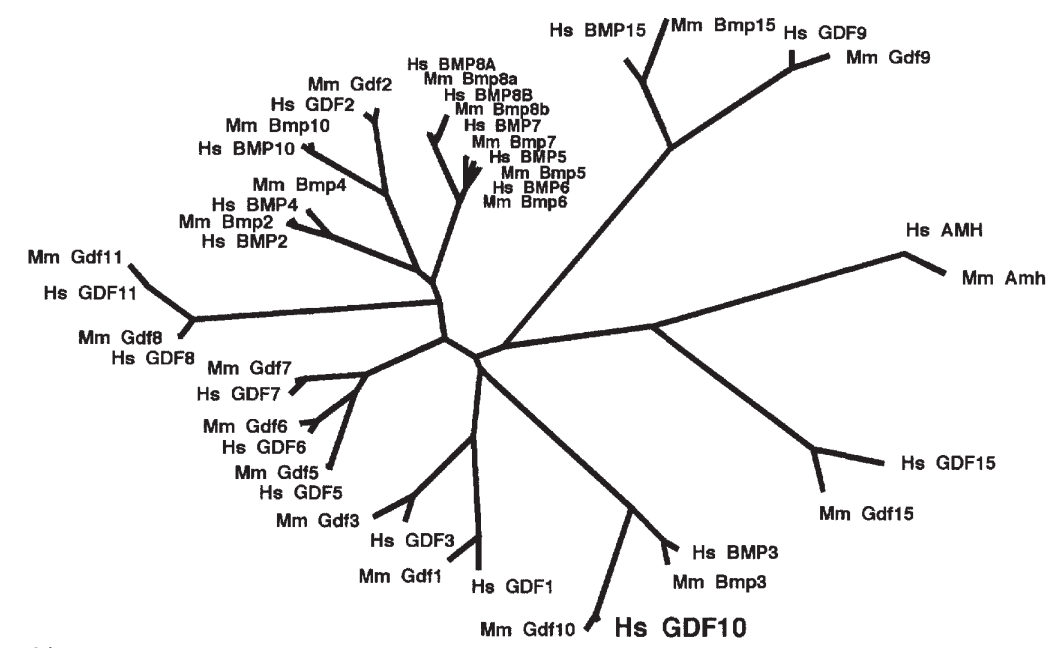

B

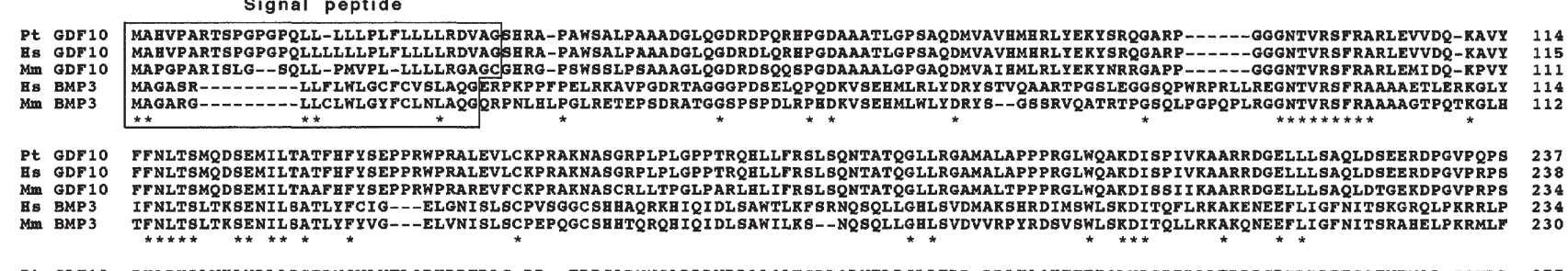

Pt GDF10 PYAPYILVYANDLAISEPNSVAVTLQR YDPFPAG-DP--EPRGAPNNSADPRVRRAAQATGPLQDNELPGLDERP-PRAHAQHFHKEQLWPSPFRALRPRPGRRDRRRKGEVFMAA-SQVLD 35

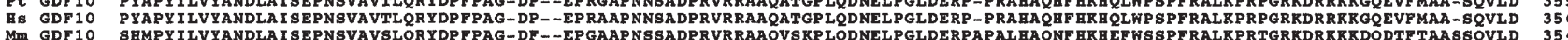

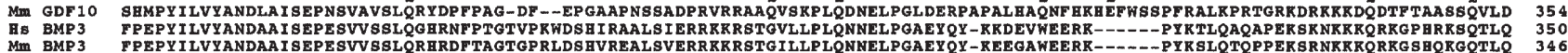

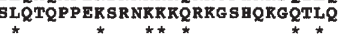
TGFB - like domain

Pt GDF10 FDEKTMQRARRRQWDEP RVCSRRYLKVDFADI GWNEWI I SPRS FDA YYCAGACEFPMPRI VRP SNHAT IQS IVRAVGI I PGI PEPCCVPDRMNSLGVLFLDESR NVVLKVYPNMSVDTCACR Hs $\operatorname{CDF} 10$ FDEKTMOAA FS BMP

Figure 2. (A), Phylogenetic analyses on BMP/GDF family. GDF10 and BMP3 are paralogs. (B), Alignment of GDF10 orthologs and BMP3 orthologs Amino-acid residues are numbered on the right. Conserved amino-acid residues are shown by asterisks. GDF10 and BMP3 orthologs are well conserved within the C-terminal TGFß-like domain (boxed).

of human $B M P / G D F$ family genes were then searched for based on manual inspection. Four TCF/LEF-binding sites were identified within the human GDF10 promoter (Fig. 1A).

Identification of the chimpanzee GDF10 gene. BLAST programs using human GDF10 RefSeq revealed that chimpanzee GDF10 gene was located within NW_112875.1 genome sequence. Exon-intron boundaries of chimpanzee GDF 10 gene were determined based on the consensus sequence of exon-intron junctions. Exon 1 corresponded to nucleotide position 152574-151803 of NW_112875.1 genome sequence, exon 2 to nucleotide position 143019-142962 and 142824-141957, and exon 3 to nucleotide position 140077139106. Chimpanzee GDF10 gene was found consisting of three exons.

Because AY412135.1 was a partial sequence derived from chimpanzee GDF10 gene, complete coding sequence (CDS) of chimpanzee GDF10 was determined by assembling nucleotide sequences of three exons in this study (Fig. 1B). Genetyx program revealed that nucleotide position 457-1890 was the coding region of chimpanzee GDF10 complete CDS (Fig. 1B). Chimpanzee GDF10 gene was found to encode a 477-aminoacid protein.

Comparative integromics analysis on mammalian BMP/GDF family members. Phylogenetic analysis on human and mouse
BMP/GDF family members revealed that GDF10 and BMP3 were paralogs (Fig. 2A). Chimpanzee GDF10 showed 99.2\%, $83.2 \%$ and $47.4 \%$ total amino-acid identity with human GDF10, mouse Gdf10 and human BMP3, respectively. Alignment of human GDF10, BMP3, chimpanzee GDF10, mouse Gdf10 and Bmp3 revealed that GDF10 and BMP3 homologs were well conserved, especially within the C-terminal TGFß-like domain (Fig. 2B).

Intra-species comparative genomics next revealed that RASGEF 1A-GDF 10-PRKG1 locus at human chromosome 10q11 and BMP3-PRKG2-RASGEF1B locus at human chromosome $4 \mathrm{q} 21$ were paralogous regions with insertions/deletions and recombination.

Expression of human GDF10 mRNA. In silico expression analyses were performed to investigate expression of human GDF 10 and mouse Gdf10 mRNAs. Human GDF10 mRNA was expressed in fetal cochlea, fetal lung, testis, retina, pineal gland, other neural tissues, head and neck tumors, while mouse Gdf10 mRNA was expressed in fetal liver, inner ear, cerebellum, other neural tissues, prostate and blood vessels.

Comparative genomics analyses on GDF10 promoters. Human GDF 10 promoter and chimpanzee GDF10 promoter were located within AL731561.12 and NW_112875.1 genome sequences, respectively, as mentioned above. BLAST programs 


\section{TCPLPZ}

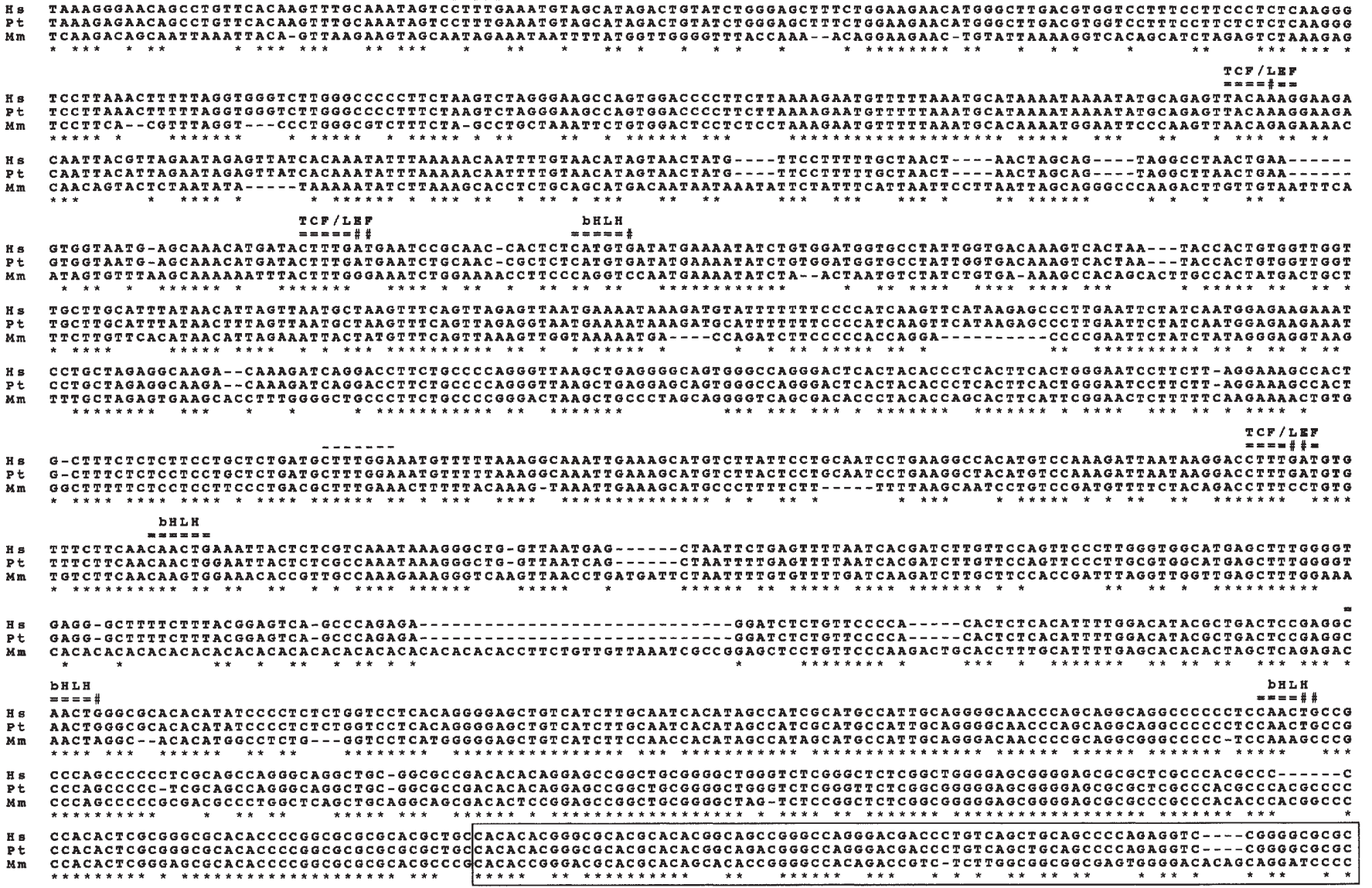

Figure 3. Mammalian GDF10 promoter. Hs, human; Pt, chimpanzee; Mm, mouse. The region corresponding to exon 1 of human GDF10 gene is boxed. Four TCF/LEF-binding sites and four bHLH-binding sites, conserved between human and chimpanzee GDF10 promoters, are shown by over-lines. Nucleotide changes to disrupt the conserved TCF/LEF- and bHLH-binding sites in mouse Gdf10 promoter are shown by a sharp. TCF/LEF-binding site in mouse Gdf10 promoter is shown by an over-line.

revealed that mouse $G d f 10$ promoter and rat $G d f 10$ promoter were located within AC166828.3 and AC106060.5 genome sequences, respectively. Because sequence gap occurred within the Gdf10 promoter region in rat AC106060.5 genome sequence, rat $G d f 10$ promoter was not used for the following comparative genomics analyses on GDF 10 promoters.

GC content of human GDF10 promoter was $53.9 \%$, that of chimpanzee GDF10 promoter was $53.8 \%$, and that of mouse Gdf10 promoter was 51.4. No significant change in the GC content was observed among mammalian GDF 10 promoters.

Four TCF/LEF-binding sites within human and chimpanzee GDF 10 promoters were not conserved in mouse $G d f 10$ promoter; however, another TCF/LEF-binding site occurred in mouse Gdf10 promoter (Fig. 3). In addition, four bHLHbinding sites within human GDF10 promoter were conserved in chimpanzee $G D F 10$ promoter, but only one in mouse $G d f 10$ promoter (Fig. 3). These facts indicate that primate GDF10 promoters and mouse Gdfl0 promoter were significantly divergent.

\section{Discussion}

TCF/LEF-binding sites within the promoter region of 22 members of $B M P / G D F$ gene family were searched for in this study. Because four TCF/LEF-binding sites were identified within human GDF 10 promoter (Fig. 1A), comparative genomics analyses on GDF10 orthologs were further performed. Chimpanzee GDF10 gene, consisting of three exons, was identified within NW_112875.1 genome sequence (Fig. 1B). AY412135.1 was not the correct coding sequence for chimpanzee GDF10. Chimpanzee GDF10 gene, encoding a 477 -amino-acid protein, showed $99.2 \%$ and $83.2 \%$ total amino-acid identity with human GDF10 and mouse Gdf10, respectively.

Phylogenetic analysis revealed that GDF10 and BMP3 were paralogs (Fig. 2A). GDF10 and BMP3 homologs were well conserved, especially within the C-terminal TGFß-like domain (Fig. 2B). Intra-species comparative genomics next revealed that RASGEF1A-GDF1-PRKG1 locus at human chromosome 10q11 and BMP3-PRKG2-RASGEF1B locus at human chromosome $4 \mathrm{q} 21$ were paralogous regions with insertions/deletions and recombination.

Human GDF10 mRNA was expressed in fetal cochlea, fetal lung, testis, retina, pineal gland, other neural tissues, head and neck tumors, while mouse Gdf10 mRNA was expressed in fetal liver, inner ear, cerebellum, other neural tissues, prostate and blood vessels. Four TCF/LEF-binding sites in human GDF 10 promoter were conserved in chimpanzee GDF 10 promoter, but not in mouse Gdf10 promoter, however, another 
TCF/LEF-binding site occurred in mouse Gdf10 promoter (Fig. 3). Four bHLH-binding sites within human GDF10 promoter were conserved in chimpanzee $G D F 10$ promoter, but only one in mouse Gdf10 promoter (Fig. 3). Primate GDF10 promoters were divergent from mouse Gdf10 promoter. Because GDF 10 was characterized as a potential target of the canonical WNT signaling pathway in neural tissues, GDF10 is one of targets of systems medicine, especially in the field of re-generative medicine.

\section{References}

1. Katoh M: $W N T$ and $F G F$ gene clusters (Review). Int J Oncol 21: 1269-1273, 2002.

2. Radtke F and Raj K: The role of Notch in tumorigenesis: oncogene or tumor suppressor? Nat Rev Cancer 3: 765-767, 2003.

3. Garciadiego-Cazares D, Rosales C, Katoh M and ChimalMonroy J: Coordination of chondrocyte differentiation and joint formation by $\alpha 5 \beta 1$ integrin in the developing appendicular skeleton. Development 131: 4735-4742, 2004.

4. Hooper JF and Scott MP: Communicating with Hedgehogs. Nat Rev Mol Cell Biol 6: 306-317, 2005.

5. Katoh Y and Katoh M: Hedgehog signaling in gastric cancer. Cancer Biol Ther 4: 1050-1054, 2005.

6. Katoh Y and Katoh M: WNT antagonist, SFRP1, is Hedgehog signaling target. Int J Mol Med 17: 171-175, 2006.

7. Varga AC and Wrana JL: The disparate role of BMP in stem cell biology. Oncogene 24: 5713-5721, 2005.

8. Katoh $\mathrm{Y}$ and Katoh M: Comparative genomics on BMP4 orthologs. Int J Oncol 27: 581-585, 2005.

9. Katoh M: Regulation of WNT signaling molecules by retinoic acid during neuronal differentiation in NT2 cells: threshold model of WNT action (Review). Int J Mol Med 10: 683-687, 2002.

10. Katoh $\mathrm{M}$ and Katoh M: Identification and characterization of human $B C L 9 L$ gene and mouse Bcl9l gene in silico. Int $\mathrm{J}$ Mol Med 12: 643-649, 2003.

11. Heller RS, Klein T, Ling Z, Heimberg H, Katoh M, Madsen OD and Serup P: Expression of WNT, Frizzled, sFRP, and DKK genes in adult human pancreas. Gene Expr 11: 141-147, 2003.

12. Katoh M: WNT2B: comparative integromics and clinical application (Review). Int J Mol Med 16: 1103-1108, 2005.

13. Katoh M: Epithelial-mesenchymal transition in gastric cancer (Review). Int J Oncol 27: 1677-1683, 2005.

14. Swain RK, Katoh M, Medina A and Steinbeisser H: Xenopus frizzled-4S, a splicing variant of Xfz4, is a context-dependent activator and inhibitor of Wnt/ß-catenin signaling. Cell Commun Signal 3: 12, 2005.

15. Katoh Y and Katoh M: Comparative genomics on FGF16 orthologs. Int J Mol Med 16: 959-963, 2005.
16. Katoh $\mathrm{M}$ and Katoh $\mathrm{M}$ : Comparative genomics on $F G F 8$, FGF17, and FGF18 orthologs. Int J Mol Med 16: 493-496, 2005.

17. Katoh $\mathrm{M}$ and Katoh M: Comparative genomics on FGF20 orthologs. Oncol Rep 14: 287-290, 2005.

18. Katoh Y and Katoh M: Comparative genomics on DKK1 orthologs. Int J Oncol 27: 275-279, 2005.

19. Katoh Y and Katoh M: Comparative genomics on DKK2 and DKK4 orthologs. Int J Mol Med 16: 477-481, 2005.

20. Heldin CH, Miyazono K and ten Dijke P: TGFß signaling from cell membrane to nucleus through SMAD proteins. Nature 390: 465-471, 1997.

21. Massague J: TGFß signal transduction. Annu Rev Biochem 67: 753-791, 1998.

22. Miyazono $\mathrm{K}$, ten Dijke $\mathrm{P}$ and Heldin $\mathrm{CH}$ : Divergence and convergence of TGFß/BMP signaling. J Cell Physiol 187: 265-276, 2001.

23. Whiteman M: Nodal signaling in early vertebrate embryos: themes and variations. Dev Cell 1: 605-617, 2001.

24. Zhang J and Li L: BMP signaling and stem cell regulation. Dev Biol 284: 1-11, 2005

25. Katoh M: Paradigm shift in gene-finding method: from benchtop approach to desk-top approach. Int J Mol Med 10: 677-682, 2002

26. Katoh $\mathrm{M}$ and Katoh $\mathrm{M}$ : Identification and characterization of human HES2, HES3, and HES5 genes in silico. Int J Oncol 25: 529-534, 2004.

27. Katoh $\mathrm{M}$ and Katoh $\mathrm{M}$ : Identification and characterization of human HESL, rat Hesl and rainbow trout hesl genes in silico. Int J Mol Med 14: 747-751, 2005

28. Katoh $\mathrm{Y}$ and Katoh M: Identification and characterization of rat Wnt6 and Wnt10a genes in silico. Int J Mol Med 15: 527-531, 2005.

29. Katoh Y and Katoh M: Comparative genomics on SLIT1, SLIT2, and SLIT3 orthologs. Oncol Rep 14: 1351-1355, 2005.

30. Katoh Y and Katoh M: Comparative genomics on HHIP family orthologs. Int J Mol Med 17: 391-395, 2006.

31. Katoh $\mathrm{Y}$ and Katoh M: Identification and characterization of rat Wnt1 and Wnt10b genes in silico. Int J Oncol 26: 841-845, 2005.

32. Katoh M and Katoh M: Comparative genomics on WNT8A and WNT8B genes. Int J Oncol 26: 1129-1133, 2005.

33. Katoh M: Molecular evolution of WNT2B orthologs. Int J Oncol 26: 1135-1139, 2005.

34. Katoh M: Comparative genomics on WNT3-WNT9B gene cluster. Int J Mol Med 15: 743-747, 2005.

35. Katoh $\mathrm{M}$ and Katoh M: Comparative genomics on WNT5A and WNT5B genes. Int J Mol Med 15: 749-753, 2005.

36. Katoh Y and Katoh M: Comparative genomics on WNT11 gene. Int J Mol Med 15: 879-883, 2005.

37. Katoh Y and Katoh M: Comparative genomics on VANGL1 and VANGL2 genes. Int J Oncol 26: 1435-1440, 2005.

38. Katoh Y and Katoh M: Comparative genomics on SFRP1 orthologs. Int J Oncol 27: 861-865, 2005. 